\title{
Distal Radius Fractures Using Acu-Loc Volar Plate
}

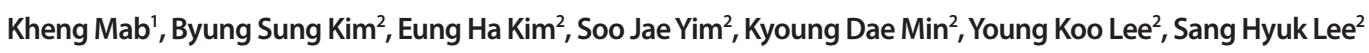 \\ 'Department of Orthopaedic Surgery, Chhlong Referal Hospital, Kratie, Cambodia; ${ }^{2}$ Department of Orthopaedic Surgery, Soonchunhyang University Bucheon \\ Hospital, Soonchunhyang University College of Medicine, Bucheon, Korea
}

\begin{abstract}
Objective: We determined the radiographic outcome of distal radius fractures with Acu-loc volar plate.
Methods: Forty-one patients were recruited between August 2009 and September 2010. There were 10 males and 31 females, with a mean age 61.2. Fractures were radiologically classification the Frykman. Fifteen fractures were group 8 , eight were group 7 , ten were group 6 , four were group 5 and four were group 3 . Distal part of the fractures was dorsally inclined in 37 wrists and inclined to volar side in four wrists. Lateral and anteroposterior radiographs taken after operated day, the fracture were compared with radiographs of the injured wrist and the differences in palmar tilt, ulnar variance; radial height; radial shift and radial inclination were measured.

Results: There was significant improvement in the measurements of radial height, radial inclination, volar tilt, ulnar variance and radial shift postoperatively. The radial height improved from an average of $8.5 \mathrm{~mm}$ (range, 3 to $15 \mathrm{~mm}$ ) to $11.0 \mathrm{~mm}$ (range, 8 to $15 \mathrm{~mm}$ ), the radial inclination improved from an average of 21.5 degree (range, 10 to 40 degree) to 28.1 degree (range, 19 to 44 degree), the palma tilt improved from an average of 12.9 degree (range, 6 to 22 degree) to 17.2 degree (range, 7 to 27 degree), the ulnar variance improved from an average of $-2.3 \mathrm{~mm}$ (range, -6 to $4 \mathrm{~mm}$ ) to $1 \mathrm{~mm}$ (range, -3 to $7 \mathrm{~mm}$ ) and the radial shift improved from an average of $18.7 \mathrm{~mm}$ (range, 15 to $26 \mathrm{~mm}$ ) to $17.3 \mathrm{~mm}$ (range, 12 to $21 \mathrm{~mm}$ ).

Conclusion: Acu-loc volar plate is a safe and effective device.
\end{abstract}

Keywords: Distal radius fractures; Acu-loc volar plate

\section{INTRODUCTION}

Distal radius fractures are the most frequently seen upper extremity fractures. Open reduction and internal fixation of distal radial fractures with an angular stable locking plate applied volarly has gained vast popularity recently [1-4]. It provides a stable fixation of osteoporotic bones, leading to anatomical restoration of the articular surface and extra articular alignment [5]. It also facilitates immediate free mobilisation of the wrist joint. However, there are few systematic studies in the literature evaluating the efficacy of locking plate fixation using objective and subjective assessments.

We have performed a prospective case study in Soonchunhyang University Hospital to document the outcomes of open reduction and internal fixation of distal radial fractures over a one year period. Today, open positioning and plate fixation are the widely rec- ognized surgical methods [6,7]. Locked plates are in the progress of replacing conventional support plates. While facilitating the positioning, those anatomical plates with screw-plate interlocking feature have more biomechanical strength against forces applied on the fracture surfaces $[8,9]$. Because of their biomechanical strength, locked plates are preferred in osteoporotic and/or multiple fractures $[6,7,10]$. However, there is no consensus neither about how to approach to distal radius nor thepositioning of the plate. During the recent years, volar approach has become more popular [8,1113]. Our objective is to study the efficacy of this method of fixation by assessing its ability to maintain radiographic reduction.

\section{MATERIALS AND METHODS}

Between August 2009 and September 2010, we treated and pro-
Correspondence to: Byung Sung Kim

Department of Orthopaedic Surgery, Soonchunhyang University Bucheon Hospital, Soonchunhyang University College of Medicine,

1174 Jung-dong, Wonmi-gu, Bucheon 420-767, Korea

Tel: +82-32-621-5262, Fax: +82-32-621-5016, E-mail: kbsos@schmc.ac.kr

Received: Jan 25, 2011 / Accepted after revision: Jun 14, 2011
(C) 2011 Soonchunhyang Medical Research Institute This is an Open Access article distributed under the terms of the Creative Commons Attribution Non-Commercial License (http://creativecommons.org/licenses/by-nc/3.0/). 
spectively evaluated the outcomes of operative treatment of $41 \mathrm{pa}-$ tients with distal radius fractures using Acu-loc volar plate (Fig. 1). Of the 41 patients, 10 were men and 31 were women, with mean age of 61.22 years (range, 34 to 81 years). Twenty-two fractures occurred on the left side and nineteen fractures on the right side. Most of the cause of injury was slipped down and road traffic accident in one patient. Fractures were radiologically classification the Frykman [14]. Fifteen fractures were group 8 (36.58\%), eight were group 7 (19.51\%), ten were group 6 (24.39\%), four were group 5 (9.75\%) and four were group 3 (9.75\%). Distal part of the fractures was dorsally inclined in 37 wrists (90.24\%) and inclined to volar side in 4 wrists $(9.75 \%)$ (Table 1$)$.

Lateral and anteroposterior radiographs taken after operated day, the fracture were compared with radiographs of the injured

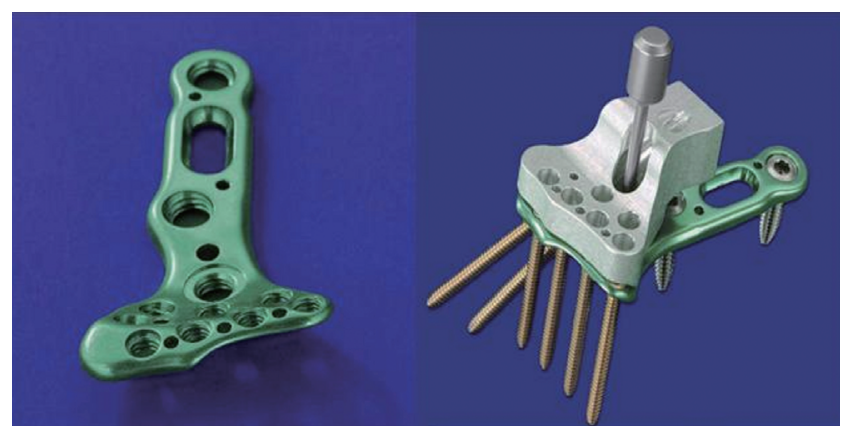

Fig. 1. Acu-loc volar plate (Acumed). wrist (Fig. 2) and the differences in palmar tilt, ulnar variance; radial height; radial shift and radial inclination were measured. Palmar tilt is the angle between a line perpendicular to the central axis of the radius and aline connecting the dorsal and palmar margins of the articular surface of the distal radius on the lateral view. It is designated as positive if the tilt is in a volar direction and nega-

Table 1. Clinical data for 41 patients with distal radius fractures treated by opened reduction using Acu-loc volar plate

\begin{tabular}{lc}
\hline Variable & Value \\
\hline Type of fracture (Frykman classification) & \\
Type 8 & $15(36.58)$ \\
Type 7 & $8(19.51)$ \\
Type 6 & $10(24.39)$ \\
Type 5 & $4(9.75)$ \\
Type 3 & $4(9.75)$ \\
Sex & \\
Female & $31(75.60)$ \\
Male & $10(24.39)$ \\
Side of fracture & \\
Right & $19(46.34)$ \\
Left & $22(53.65)$ \\
Age lyr) & $61.22(34-81)$ \\
Displace of fracture & \\
Volar & $4(9.75)$ \\
Dorsally & $37(90.24)$ \\
\hline
\end{tabular}

Values are presented as number (\%) or mean (range).
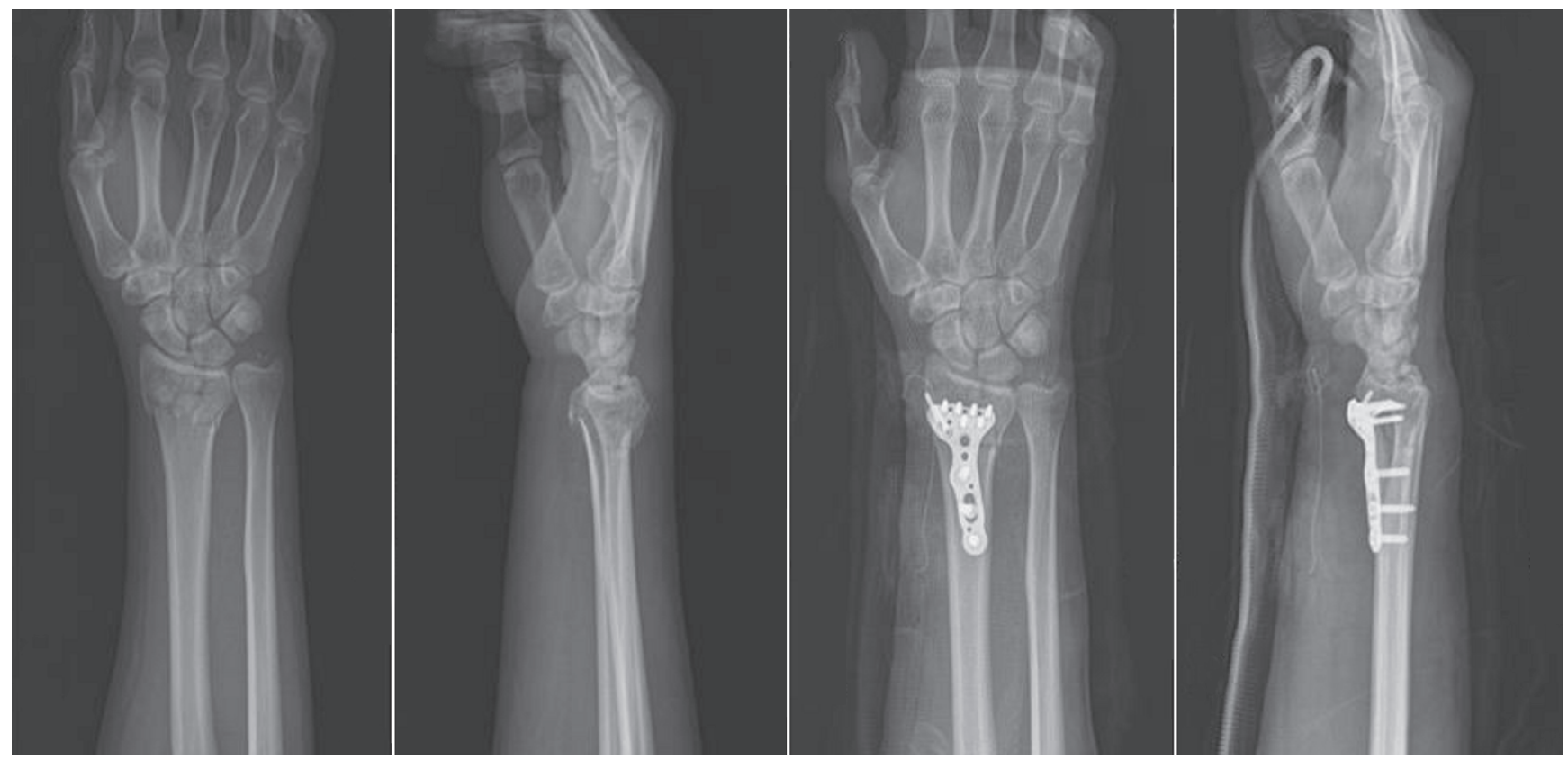

Fig. 2. Lateral and anteroposterior radiographs of the injured wrist type 8 according to Frykman and after operated by using Acu-loc volar plate. 
tive if there is a dorsal tilt. Ulnar variance and radial inclination were measured on anteroposterior radiographs. Ulnar variance is the distance in millimeters between two parallel lines perpendicular to the central axis of the radius, one line passing through the distal articular surface of the ulna and the other through the medial articular surface of the distal radius. Ulnar variance is negative when the ulnar articular surface is more proximal with respect to the radial articular surface and positive if it is more distal. This is an accurate indication of the degree of radial shortening. Radial inclination is the angle between a line perpendicular to the central axis of the radius and the line connecting the radial and ulnar limits of the articular surface of the distal radius. Radial height is measured on an anteroposterior (AP) radiograph. It is the distance between two lines perpendicular to the long axis of the distal radius; the first one going through the tip of the styloid process, the second one at the level of the articular surface of the ulnar head. Radial width is defined as the distance between two lines parallel to the longitudinal axis, one going through the most lateral tip of the styloid process and one going through the centre of the radius on an AP radiograph. An increased radial width as compared with the non-injured side is a strong indication for rotational malalignment and as such a predictor of bad functional outcome. Postoperative radiographs were taken at every visit. These were scheduled the weeks after surgery, at 2 , at 4 to 6 weeks after surgery, at monthly intervals as needed.

The mean pre-surgical period was 7.82 days (range, 2 to 26 days). The operating time was 41.09 minutes (range, 20 to 65 minutes), and the hospital stay was 4.92 days (range, 2 to 10 days).

\section{Surgical Technique}

Surgery was performed under general anesthesia with an arm tourniquet. The patient's forearm is supinated to expose the surgical site. To maximize exposure, a towel is positioned under the wrist placing it in extension. Make a longitudinal incision approximately six centimeters in length just radial to the flexor carpi radialis tendon to protect against injury to the palmar cutaneous branch of the median nerve. The tendon sheath is opened and the tendon is retracted radially to protect the radial artery. The flexor pollicus longus is identified by passive flexion/extension of the thumb interphalangeal joint and is retracted ulnarly to protect the median nerve. Next, the pronator quadratus is identified by its transverse fibers and is released radial to ulnar to expose the fracture site. The fracture is reduced and evaluated under fluoroscopy. The brachio- radialis may need to be released from its insertion on the radial styloid to facilitate reduction and visualization of the fracture. The plate is designed to sit along the distal aspect of the radius to support the volar articular fracture fragments. Once the appropriate plate is selected, attach the corresponding targeting guide using the set screw (80-0038). This may be done on the back table prior to insertion. The plate's position is then secured proximally with a $.045^{\prime \prime} \mathrm{K}$-wire and distally with a .054" K-wire. If the targeting guide is not already attached to the plate, you would then slide the guide over the distal K-wire and into position. Another method is to secure the plate to the bone with a cortical screw proximally and then attach the targeting guide. Place the first $3.5 \mathrm{~mm}$ non-locking cortical screw through the slot in the plate. The position of the plate relative to the articular surface can then be fine tuned by sliding the plate proximal or distal under fluoroscopy. Using the $2.8 \mathrm{~mm}$ drill (MS-DC28) and the drill guide (PL-2018), drill through the far cortex. Drill depth is measured with the depth gauge (MS-9020). Note that if provisional K-wires are in place, they may interfere with drilling and screw insertion. Insert the appropriate silver $3.5 \mathrm{~mm}$ non-locking screw $(\mathrm{CO}-3 \times x 0)$, taking care that the screw is the proper length. The screw may need to be downsized after the plate has been reduced down to the bone. To assess the position of the distal locking screws relative to the articular surface and the dorsum of the radius, a .054" K-wire may be placed through the distal holes on the targeting guide and plate. The fracture reduction, plate position, and the location of the K-wire relative to the joint is assessed under fluoroscopy. If the distal K-wires do not penetrate the joint, the distal $2.3 \mathrm{~mm}$ screws will not either. Care should be taken not to angle the distal K-wires. Target one of the four distal holes first. Insert the drill guide (MS-DG23) into one of the holes, followed by the $2.0 \mathrm{~mm}$ drill (MS-DCR20). Screw length is measured by using the laser mark on the drill and the scale on the drill guide. As an alternative, the depth probe (MS-DRPB) may be used by hooking the far cortex and measuring with the laser mark on the probe. There are three types of $2.3 \mathrm{~mm}$ screws that can be used in any of the eight distal holes: fully-threaded locking screws (gold), smooth locking pegs (bronze) and non-toggling screws (silver). All $2.3 \mathrm{~mm}$ screws are inserted using the $1.5 \mathrm{~mm}$ driver tip (HPC0015), sleeve (MS-SS23) and driver handle (MS-2210). It is at the discretion of the surgeon when to use the threaded locking screws, the smooth locking pegs, and the non-toggling (non-locking) screws. The thread pitch on the threaded locking screw is the same from the tip to the head minimizing the "differential pitch effect" as the 
screw is seated into the plate. All eight distal holes accept the three different screw designs. The radial styloid screws are designed specifically to target and support the radial styloid fragment at angles of 41 and 53 degrees from the plate. A C-Arm overlay is available in the system to determine the trajectory of the distal/radial screw prior to screw insertion. The overlay is used with an AP view of the distal radius. The two radial styloid screws are approached from the back of the targeting guide. Using the dual slot on the back of the guide, the distal/radial screw is targeted by inserting the drill guide to the radial side of the dual slot. The more proximal/ulnar screw is targeted by inserting the drill guide to the ulnar side of the dual slot. Both radial styloid screws should be drilled through the targeting guide. Remove the guide to measure and insert the screws. The guide is removed to increase visualization of the drill holes when inserting the screws. With the targeting guide in place, it may be difficult to remove the radial styloid screws if a different size screw is needed. If resizing is necessary, remove the guide and the screw, measure with the depth gauge and insert the proper screw. Select one of the two remaining proximal holes and insert the threaded drill guide (MS-LDG35). Drill with the $2.8 \mathrm{~mm}$ drill (MS-DC28) and measure with the depth gauge (MS-9020). Insert the proper length $3.5 \mathrm{~mm}$ light blue locking screw (COL-3XX0) using the $2.5 \mathrm{~mm}$ driver tip (HPC-0025), sleeve (MS-SS35) and driver handle (MS-3200), taking care that the screw does not exit the bone dorsally. Using the same process, drill and place the final locking screw. Following thorough radiographic evaluation, check alignment and rotation, then close. Start immediate finger range of motion and forearm rotation post-operation. Allow early functional use of the hand for light adult daily livings. Support the wrist according to bone quality and stability.

\section{RESULTS}

There were thirty-one females and ten males with a mean age of 61.22 years (range, 34 to 81 years). Twenty-two fractures occurred on the left side and nineteen fractures on the right side. Most of the cause of injury was slipped down and road traffic accident in one patient. Fractures were radiologically classification the Frykman. Fifteen fractures were group 8 (36.58\%), eight were group 7 (19.51\%), ten were group $6(24,39 \%)$, four were group 5 (9.75\%) and four were group 3 (9.75\%). The mean pre-surgical period was 7.82 days (range, 2 to 26 days). The operating time was 41.09 minutes (range, 20 to 65 minutes), and the hospital stay was 4.92 days (range, 2 to 10 days). There was a significant improvement in the measurement of palmar tilt, ulnar variance; radial height; radial shift and radial inclination after opened reduction and internal fixation using Acu-loc volar plate. The measurement of the two intervals were stationary. The radial height improved from an average of $8.56 \mathrm{~mm}$ (range, 3 to $15 \mathrm{~mm}$ ) to $11.04 \mathrm{~mm}$ (range, 8 to $15 \mathrm{~mm}$ ), the radial inclination improved from an average of 21.53 degree (range, 10 to 40 degree) to 28.12 degree (range, 19 to 44 degree), the palma tilt improved from an average of 12.92 degree (range, 6 to 22 degree) to 17.24 degree (range, 7 to 27 degree), the ulnar variance improved from an average of $-2.31 \mathrm{~mm}$ (range, -6 to $4 \mathrm{~mm}$ ) to $1 \mathrm{~mm}$ (range, -3 to $7 \mathrm{~mm}$ ) and the radial shift improved from an average of $18.78 \mathrm{~mm}$ (range, 15 to $26 \mathrm{~mm}$ ) to $17.36 \mathrm{~mm}$ (range, 12 to $21 \mathrm{~mm}$ ) (Table 2).

\section{DISCUSSION}

Useful parameters to determine whether the anatomy of a fracture indicates instability and will need surgical stabilization are: excessive comminution initial loss of $15 \mathrm{~mm}$ or more of radial length initial dorsal tilt of $20^{\circ}$ or more $[15,16]$. Surgical intervention becomes an important consideration when an acceptable reduction can not be either achieved or maintained with cast immobilization. Post-operative radiographic measurements demonstrate that joint congruity was restored to normal in all. The extra-articular parameters including palmar tilt, radial height and inclination were improved uniformly. These radiographic improvements were maintained in all.

Locked plates that are widely used provide successful results es-

Table 2. Distal radial measurements as determined by radiography at the two intervals (pre-operative, post-operative)

\begin{tabular}{|c|c|c|c|c|c|}
\hline Measurement interval & Radial height (mm) & Radial inclination (degree) & Volar tilt (degree) & Ulnar variance (mm) & Radial shift (mm) \\
\hline Pre-operative & $8.56(2.61)$ & $21.54(6.94)$ & $13.93(4.18)$ & $-2.34(2.60)$ & $18.78(2.34)$ \\
\hline Post-operative & $11.05(1.79)$ & $28.12(5.37)$ & $17.24(4.56)$ & $1(2.24)$ & $17.37(1.74)$ \\
\hline
\end{tabular}

Values are presented as mean (SD).

Differences between pre-operative and post-operative for radial height, radial inclination, volar tilt, ulnar variance and radial shift were significant. 
pecially for the treatment of intraarticular unstable fractures of distal radius $[1,6,10,13,17]$. This method, which is effective in anatomic realignment, allows early joint motion, owing to its fixation strength $[18,19]$. Close placement to joint interface and screwing capability in different orders are its biomechanical superiorities. Volar approach provides both access with minimal surgical trauma on distal radius and fixation with a better adaptation to surrounding tissues [6-8,10,12,17,20,21]. In the subjects of our study, a successful anatomic alignment was acquired with volar approach, regardless of the direction of fracture angulation. The ability of locked screws to resist the loads in the distal radius has been shown in several studies that compared the average construct failure load of several plates on the market. Acumed simulated the testing methods used in these studies to determine the failure load of the Aculoc plate. The failure load of the Acu-loc plate was compared with the results of two recent biomechanical studies. In the first study, the biomechanical properties of six dorsal and volar plate designs were compared [22]. Average construct failure load of the six plates was measured. The study stated that an estimated $250 \mathrm{~N}$ is the amount of force that is applied to the wrist joint in the flexed digit position. Testing conducted on the Acu-loc plate resulted in a construct load of 2,400 N without failure. This shows that the Acu-loc can withstand nearly 9X the force that is applied to the wrist during patient rehabilitation. All plates, including the Acu-loc, exceeded this $250 \mathrm{~N}$ benchmark. The six plates in the study failed in a similar fashion. Bending of the plates occurred without screw loosening. The Acu-loc's biomechanical results were also compared to the results of a second biomechanical study [23]. In this study, the average construct failure load of three volar plate designs were compared. Screw loosening and bending occurred at the point of failure for the three plates studied.

In conclusion, we believe that, our study would provide additional knowledge and experience about the treatment of distal radius fractures. As a result, Acu-loc volar plates are effective in the correction and maintenance of distal radius anatomy. By using those plates, joint motion and daily functioning is recovered in a shorter time.

\section{ACKNOWLEDGEMENTS}

I should like to acknowledge my gratitude, with my warmest regard, to professor Won Han Shin, former director of Soonchunhyang University Bucheon Hospital, Neurosurgical professor and professor Dea Shik Hong director of Soonchunhyang University Bucheon Hospital whose help, encouragement and stimulating suggestions have helped me greatly as well as all Cambodian doctors studying here in all time of research and writing of this presentation.

I would like to express my gratitude to those who given me the opportunities to complete this presentation: my special thanks go to professor Eung Ha Kim, professor Soo Jae Yim, professor Kyoung Dae Min, professor Byung Sung Kim, Young Koo Lee, and Sang Hyuk Lee.

I want to thank the Department of Orthopedic Surgery of Soonchunhyang, Bucheon, for giving me permission to commence this presentation, to do the necessary research work and to use department data.

\section{REFERENCES}

1. Arora R, Lutz M, Fritz D, Zimmermann R, Oberladstätter J, Gabl M. Palmar locking plate for treatment of unstable dorsal dislocated distal radius fractures. Arch Orthop Trauma Surg 2005;125:399-404.

2. Chen NC, Jupiter JB. Management of distal radial fractures. J Bone Joint Surg Am 2007;89:2051-62.

3. Osada D, Kamei S, Masuzaki K, Takai M, Kameda M, Tamai K. Prospective study of distal radius fractures treated with a volar locking plate system. J Hand Surg Am 2008;33:691-700.

4. Othman AY. Fixation of dorsally displaced distal radius fractures with volar plate. J Trauma 2009;66:1416-20.

5. Leung F, Tu YK, Chew WY, Chow SP. Comparison of external and percutaneous pin fixation with plate fixation for intra-articular distal radial fractures. A randomized study. J Bone Joint Surg Am 2008;90:16-22.

6. Simic PM, Weiland AJ. Fractures of the distal aspect of the radius: changes in treatment over the past two decades. Instr Course Lect 2003;52:18595.

7. Trumble TE, Culp RW, Hanel DP, Geissler WB, Berger RA. Intra-articular fractures of the distal aspect of the radius. Instr Course Lect 1999;48: 465-80.

8. Willis AA, Kutsumi K, Zobitz ME, Cooney WP 3rd. Internal fixation of dorsally displaced fractures of the distal part of the radius. A biomechanical analysis of volar plate fracture stability. J Bone Joint Surg Am 2006;88: 2411-7.

9. Levin SM, Nelson CO, Botts JD, Teplitz GA, Kwon Y, Serra-Hsu F. Biomechanical evaluation of volar locking plates for distal radius fractures. Hand (N Y) 2008;3:55-60.

10. Orbay JL, Touhami A. Current concepts in volar fixed-angle fixation of unstable distal radius fractures. Clin Orthop Relat Res 2006;445:58-67.

11. Khanduja V, Ng L, Dannawi Z, Heras L. Complications and functional outcome following fixation of complex, intra-articular fractures of the distal radius with the AO Pi-Plate. Acta Orthop Belg 2005;71:672-7.

12. Musgrave DS, Idler RS. Volar fixation of dorsally displaced distal radius fractures using the 2.4-mm locking compression plates. J Hand Surg Am 2005;30:743-9.

13. Rozental TD, Blazar PE. Functional outcome and complications after volar plating for dorsally displaced, unstable fractures of the distal radius. J 
Hand Surg Am 2006;31:359-65.

14. Frykman G. Fracture of the distal radius including sequelae--shoulderhand-finger syndrome, disturbance in the distal radio-ulnar joint and impairment of nerve function. A clinical and experimental study. Acta Orthop Scand 1967:Suppl 108:3+.

15. Nana AD, Joshi A, Lichtman DM. Plating of the distal radius. J Am Acad Orthop Surg 2005;13:159-71.

16. Goldfarb CA, Yin Y, Gilula LA, Fisher AJ, Boyer MI. Wrist fractures: what the clinician wants to know. Radiology 2001;219:11-28.

17. Ruch DS, Papadonikolakis A. Volar versus dorsal plating in the management of intra-articular distal radius fractures. J Hand Surg Am 2006;31:916.

18. Knirk JL, Jupiter JB. Intra-articular fractures of the distal end of the radius in young adults. J Bone Joint Surg Am 1986;68:647-59.
19. Chung KC, Watt AJ, Kotsis SV, Margaliot Z, Haase SC, Kim HM. Treatment of unstable distal radial fractures with the volar locking plating system. J Bone Joint Surg Am 2006;88:2687-94.

20. Kamano M, Honda Y, Kazuki K, Yasuda M. Palmar plating for dorsally displaced fractures of the distal radius. Clin Orthop Relat Res 2002;(397): 403-8.

21. Murakami K, Abe Y, Takahashi K. Surgical treatment of unstable distal radius fractures with volar locking plates. J Orthop Sci 2007;12:134-40.

22. Osada D, Viegas SF, Shah MA, Morris RP, Patterson RM. Comparison of different distal radius dorsal and volar fracture fixation plates: a biomechanical study. J Hand Surg Am 2003;28:94-104.

23. Osada D, Fujita S, Tamai K, Iwamoto A, Tomizawa K, Saotome K. Biomechanics in uniaxial compression of three distal radius volar plates. J Hand Surg Am 2004;29:446-51. 